\title{
Ultrafast imaging of transient electronic plasmas produced in conditions of femtosecond waveguide writing in dielectrics
}

\author{
W. Gawelda, ${ }^{\text {a) }}$ D. Puerto, J. Siegel, A. Ferrer, A. Ruiz de la Cruz, H. Fernández, and \\ J. Solis ${ }^{\text {b) }}$ \\ Laser Processing Group, Instituto de Óptica, C.S.I.C., Serrano 121, 28006 Madrid, Spain
}

(Received 7 July 2008; accepted 1 September 2008; published online 24 September 2008)

\begin{abstract}
Femtosecond laser-induced plasmas in bulk dielectrics are imaged under waveguide writing conditions, for different polarizations, pulse durations, and processing depths, and their temporal evolution is measured using ultrafast pump-probe microscopy. The irradiation beam profile is elliptically shaped yielding a disklike focal volume. We demonstrate for doped phosphate glass that increasing the pulse duration improves the spatial distribution of deposited energy by minimizing beam filamentation and prefocal depletion effects. As a consequence, energy deposition in the desired volume is greatly enhanced. Our results identify key parameters for optimizing femtosecond laser processing of dielectrics and different strategies to minimize energy loss channels. () 2008 American Institute of Physics. [DOI: 10.1063/1.2988275]
\end{abstract}

The use of femtosecond (fs) laser pulses for nonlinear processing of dielectrics has opened new possibilities for fabrication of active and passive integrated photonics devices. ${ }^{1}$ In particular Er:Yb-doped phosphate glasses were recognized as suitable materials for fabrication of compact mode-locked waveguide lasers by direct laser writing. ${ }^{2}$ This technique explores nonlinear interaction of the pulse with the material leading to localized multiphoton absorption and avalanche ionization in the focal region, ${ }^{3}$ creating a dense electron plasma. This short-lived plasma may trigger a permanent refractive index change in a spatially confined region. ${ }^{1}$ Yet, nonlinear effects, such as self-focusing ${ }^{4}(\mathrm{SF})$ and related beam filamentation ${ }^{5}(\mathrm{BF})$ can severely compromise the efficient and localized deposition of laser energy in the desired volume. Therefore an effective control of the spatio-temporal properties of fs laser-induced transient plasmas is paramount for the production of optimized photonic elements. Femtosecond pump-probe microscopy allows imaging both the temporal and spatial evolution of transient changes in the optical properties of a material upon irradiation with ultrashort laser pulses. ${ }^{6}$ This method has been successfully applied for studying surface and bulk material interactions.

Here, time-resolved images of spatial distributions of laser-induced plasmas and transient refractive index changes are recorded under real processing conditions by means of fs pump-probe microscopy and time-integrated plasma emission microscopy in commercial phosphate glass doped with $2.2 \% \mathrm{Er}^{3+}$ and $2.5 \% \mathrm{Yb}^{3+}$ (wt. \%) (MM2 from Kigre Inc.). We have simultaneously applied elliptical beam shaping ${ }^{9}$ and pulse chirping ${ }^{10}$ in order to control the spatio-temporal profile of the fs laser pulse used for waveguide writing in doped phosphate glass. Spatial beam shaping is accomplished by using a horizontal slit inserted in the pump beam. ${ }^{11}$ We have also investigated the influence of laser polarization and processing depth on the properties of transient plasmas in relation to SF and BF phenomena.

Our experimental setup is shown in Fig. 1(a). It is based

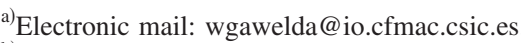

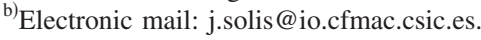

on a pump-probe geometry using a single laser pulse selected from the pulse train by means of an electromechanical shutter. A major fraction of an $\sim 100 \mathrm{fs}, 800 \mathrm{~nm}$ laser pulse (pump) is focused inside the sample using a microscope objective $(\mathrm{MO}) \quad[10 \times$, numerical aperture $(\mathrm{NA})=0.26]$, whereas the remaining low-intensity beam (probe) illuminates the focal region at a variable time delay with respect to the pump pulse. The transmitted probe beam is sent to a 12 bit CCD camera using a combination of MO $(20 \times$, NA $=0.42)$ and a tube lens, effectively imaging the focal region of the pump beam. The time-zero delay $(\Delta t=0 \mathrm{ps})$ corresponds to the appearance of the transient signal within the field of view of the images recorded. An additional interfer-

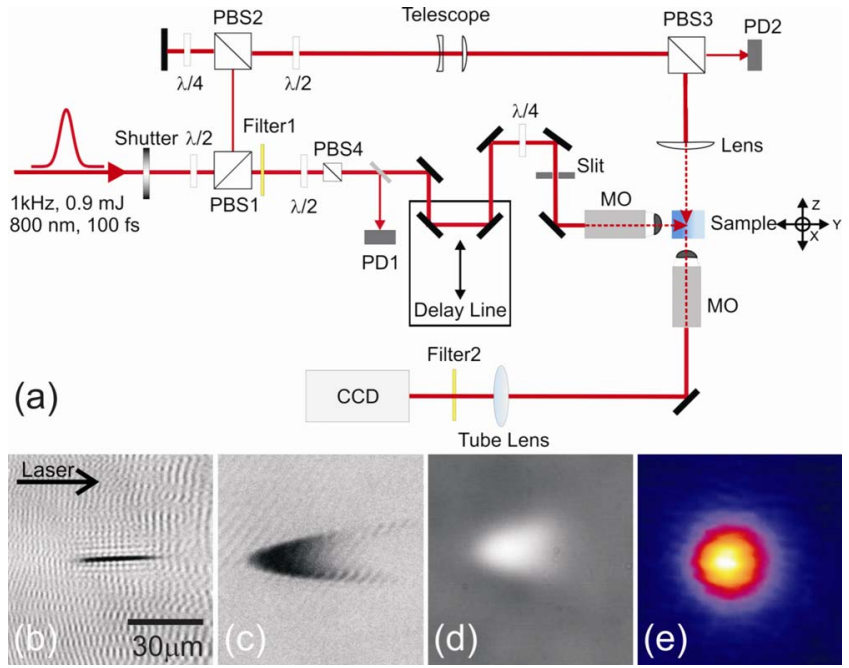

FIG. 1. (Color online) Schematic layout of a fs pump-probe microscope. (a) Polarizing beamsplitters (PBS) are used to split the laser beam into pump and probe arms (PBS1) and vary the pump energy (PBS4 and a $\lambda / 2$ plate) measured with a photodiode PD1. PBS2 and PBS3 are used to adjust probe beam polarization for sample illumination in reflectivity mode (not discussed here) and beam diagnostics (PD2). Filter1 is a long-pass IR filter. Time-resolved transmission image of the spatial plasma distribution recorded in fused silica for a time delay of $\Delta t=0.2 \mathrm{ps}$ without the slit (b) and with a $0.35 \mathrm{~mm}$ horizontal slit width (c) at the processing depth of $1.45 \mathrm{~mm}$ with pulse energies of 0.25 and $6 \mu \mathrm{J}$, respectively. (d) Transillumination image of a femtosecond laser-written waveguide under similar conditions as in (c) and the corresponding guided mode at $633 \mathrm{~nm}(\mathrm{e})$. 


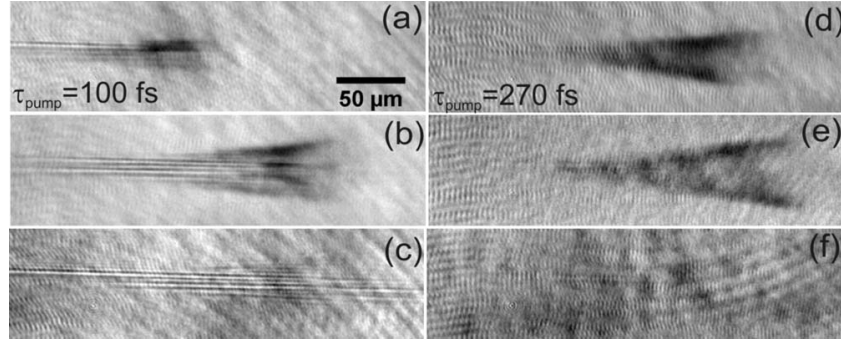

FIG. 2. Transient transmission images recorded in bulk Er:Yb codoped phosphate glass at $1.56 \mathrm{~mm}$ depth with circularly polarized TL pump pulses of $8 \mu \mathrm{J}$ energy (at the sample site) [(a)-(c)] and stretched pulses $\tau_{\text {pump }}$ $=270 \mathrm{fs}[(\mathrm{d})-(\mathrm{f})]$ at several time delays $\Delta t$, i.e., $0.65 \mathrm{ps}$ in (a) and (c), $1.2 \mathrm{ps}$ for (b) and (e), and 50 ps for images (c) and (f). In all images the laser beam impinges from the left.

ence bandpass filter (Filter2) centered at $800 \mathrm{~nm}(10 \mathrm{~nm}$ bandwidth) removes the plasma emission contribution to the transmitted probe signal. The plasma emission spectrum consists of a broad emission band in $\sim 400-650 \mathrm{~nm}$ range (data not shown here). In order to reproduce waveguide writing conditions, as in Refs. 9 and 10, where $100 \mu \mathrm{m} / \mathrm{s}$ writing speed was used, the fresh sample was preirradiated with 19 pulses before a time-resolved image corresponding to the 20th pulse was recorded at a given time delay and the sample was moved to a fresh region. Time-resolved images in Figs. 1(b) and 1(c) show the effect of slit shaping in bulk fused silica, which was used as a reference sample for setup optimization. In Fig. 1(b), for time delay $\Delta t=0.2 \mathrm{ps,} \mathrm{the} \mathrm{plasma}$ distribution (dark region) shows the expected elongated cross section due to both the low NA of the focusing optics and a small contribution of spherical aberrations induced at the corresponding processing depth. ${ }^{9}$ By introducing a slit (0.35 mm width), the focus is expanded transversely to the beam propagation axis, yielding a disk-shaped focal volume. The corresponding shape of the transient plasma distribution, additionally affected by SF, can be seen in Fig. 1(c). The shape of the light guiding region, i.e., Figs. 1(d) and 1(e), where a local increase in the refractive index is detected, coincides with that of the plasma absorption observed in Fig. $1(\mathrm{c})$.

We have applied this technique to study the laserinduced plasma and filamentation dynamics in an application-relevant material such as Er:Yb-doped phosphate. In earlier reports, it has been shown that temporally shaped femtosecond laser pulses can significantly improve the energy coupling in dielectrics ${ }^{12}$ and lower the thresholds for ionization processes. ${ }^{13}$ Here, we have adjusted the laser amplifier compressor settings to stretch the pulse duration via linear pulse chirping. Figures 2(a)-2(c) show timeresolved transmission images recorded in doped phosphate glass irradiated with a transform-limited (TL) pulse with a duration $\tau_{\text {pump }} \sim 100 \mathrm{fs}$ (full width at half maximum). The free electron plasma shows the characteristic arrow head shape (as previously observed in fused silica), as shown in Figs. 2(a) and 2(b). In order to estimate the transient electron densities $n_{e}$ of these plasmas, we have applied the Drude model $^{14}$ and transmission values extracted from timeresolved images. Our results yield the transient electronic density in the focus to be $n_{e} \approx 2 \times 10^{21} \mathrm{~cm}^{-3}$, which is below the damage threshold in terms of plasma density, i.e., $n_{e}$ $<n_{d}\left(\approx 7 \times 10^{21} \mathrm{~cm}^{-3}\right.$ for Er: $\mathrm{Yb}$ codoped phosphate glass) but above the critical density $n_{c}$ at $800 \mathrm{~nm}\left(\approx 1 \times 10^{21} \mathrm{~cm}^{-3}\right)$.

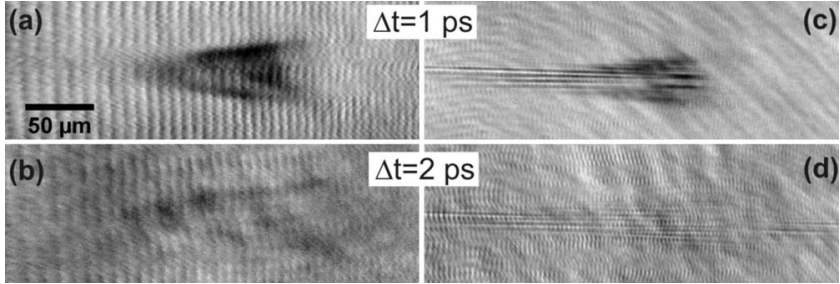

FIG. 3. Time-resolved spatial distributions of laser-induced plasmas with circularly polarized TL pump pulses as a function of the processing depth. The pump pulse energy was fixed to $8 \mu \mathrm{J}$ at the sample site. The absence of filamentation at shallow depths, i.e., $0.62 \mathrm{~mm}$, is shown in (a) and (b) at different time delays $\Delta t$. Multiple filaments are clearly visible at $1.56 \mathrm{~mm}$ depth in (c) and (d). In all images the laser beam impinges from the left.

In addition, we observe transient transmission changes caused by multiple BF in the prefocal region. Multiple filamentation is a direct consequence of the input beam ellipticity. ${ }^{15}$ The filaments observed in Figs. 2(a) and 2(c) are already present at the earliest time delays $(\Delta t=0.1 \mathrm{ps})$ and their effect on the transient transmission changes persists up to $1 \mathrm{~ns}$ (not shown). To our knowledge the existence of filament-induced transient changes up to such long delays has not been observed so far in bulk dielectrics. We estimate the intensity in the filaments ${ }^{4} \quad I_{f}=P_{\text {crit }} / \pi\left(r_{f}\right)^{2}=3.64$ $\times 10^{12} \mathrm{~W} / \mathrm{cm}^{2}$, where $P_{\text {crit }}=\pi(0.61)^{2} \lambda_{0} / 8 n_{0} n_{2}=1.03 \mathrm{MW}$ denotes critical power for SF for linearly polarized laser beam in Er:Yb-doped phosphate glass (with $n_{0}$ and $n_{2}$ being its linear and nonlinear refractive indices, respectively) at $800 \mathrm{~nm}$, and $r_{f} \approx 3 \mu \mathrm{m}$ is the filament radius measured from the time-resolved images. In Figs. 2(d)-2(f), the material response upon interaction with a stretched pump pulse, i.e., $\tau_{\text {pump }}=270 \mathrm{fs}$, is shown. The stronger and spatially broader transient free electron plasma is a clear indication of an improved energy coupling in the focal region of the stretched laser pulse. The most striking difference is the complete absence of filamentation in the latter case, most likely due to the reduced peak power of the pulse. The comparison of the results observed for both pulse durations, clearly shows that both prefocal beam energy depletion caused by $\mathrm{SF}^{16}$ and the formation of multiple filaments ${ }^{15,17}$ act as a very efficient energy loss channels that limit the amount of energy deposited in the focal region.

Since SF and the related nonlinear BF are inherently bound to nonlinear propagation in a Kerr medium, a substantial change in the observed plasma evolution is expected at different processing depths. Figures 3(a)-3(d) show images recorded for a TL pulse $\left(\tau_{\text {pump }}=100 \mathrm{fs}\right)$ at $0.62 \mathrm{~mm}$ and $1.56 \mathrm{~mm}$ depth, respectively. Indeed, filamentation is strongly enhanced at larger depths, although the overall spatial profile of the plasma is rather similar in both cases. Similarly, laser polarization influences the BF process by modifying the SF critical power $P_{\text {crit }}$ via the polarizationdependent coefficient $n_{2}$. The ratio of nonlinear refractive indices for linear $\left(n_{2}{ }^{\perp}\right)$ and circular $\left(n_{2}{ }^{o}\right)$ polarizations in an isotropic medium scale as $n_{2}{ }^{\perp} / n_{2}{ }^{o}=1.5{ }^{18}$ The values of $n_{2}{ }^{\perp}=5.85 \times 10^{-20} \mathrm{~m}^{2} / \mathrm{W}$ and $n_{2}{ }^{o}=3.94 \times 10^{-20} \mathrm{~m}^{2} / \mathrm{W}$, for Er:Yb-doped phosphate glass, were measured using femtosecond degenerate four wave mixing. ${ }^{19}$ The experimentally obtained ratio of $n_{2}{ }^{\perp} / n_{2}{ }^{o}=1.49$ is in excellent agreement with theory and leads to a ratio of critical powers $P_{\text {crit }}{ }^{\perp} / P_{\text {crit }}{ }^{o}=n_{2}{ }^{o} / n_{2}{ }^{\perp}=0.67$. 


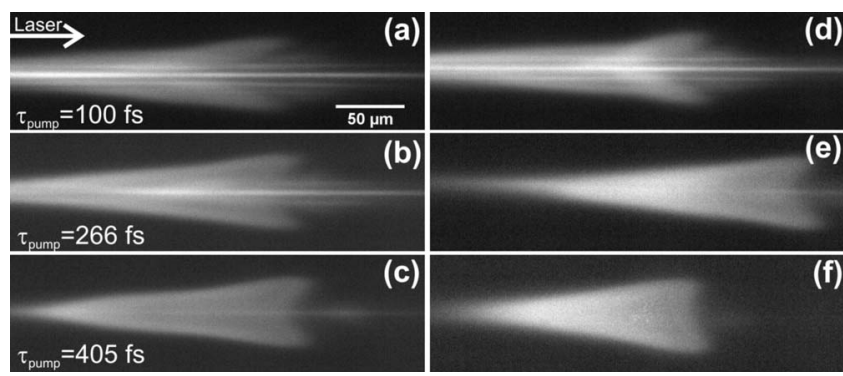

FIG. 4. Plasma emission images recorded for various pump pulse durations $\tau_{\text {pump }}$ under the same experimental conditions, i.e., pump energy, processing depth. In (a)-(c) plasma emission images for horizontal polarization are shown, whereas (d)-(f) present plasma emission images for circularly polarized laser with the same values of $\tau_{\text {pump }}$ as in (a)-(c).

The strong influence of laser polarization on the onset of filamentation can be seen in the plasma emission images for both polarization states of the pump pulse and increasing pump pulse durations in Fig. 4. These images were recorded by blocking the probe beam and removing Filter 2 and correspond to an average of six consecutive pump pulses. The main difference between the time-resolved transmission and plasma emission images is the ability of the former to capture both temporal and spatial properties of the laser-induced plasmas in "real time." Plasma emission allows only timeintegrated optimization of the spatial distribution of the observed plasmas. As already seen in Fig. 2, filamentation is efficiently reduced as we increase the pulse duration. However, only for circularly polarized laser beam [see Figs. 4(d)-4(f)] filamentation is fully suppressed, while residual filaments for horizontal polarization [Fig. 4(c)] are still present even at the longest pulse duration $\tau_{\text {pump }}=405 \mathrm{fs}$. The more efficient reduction in filamentation using circular polarization is consistent with the concomitant increase from $P_{\text {crit }}{ }^{\perp}=1.03 \mathrm{MW}$ to $P_{\text {crit }}{ }^{o}=1.53 \mathrm{MW}$, as discussed above. The importance of laser polarization in multiphoton ionization mechanisms in dielectrics has been recognized earlier. ${ }^{20}$ Particularly in fs laser waveguide writing, ${ }^{21}$ waveguides produced with circularly polarized light show lower propagation losses when compared to linear polarization. The authors attributed this improvement to the absence of periodic nanostructures; formed in various glasses upon irradiation with linearly polarized ultrashort laser pulses. ${ }^{22,23}$ Our findings suggest that circular polarization not only avoids the generation of nanofractures but also minimizes BF effects and improves the spatial distribution of the transient plasma, which directly leads to better quality of the laser-written structures. Therefore we believe these findings are of fundamental relevance to many applications where fs laser pulses are employed for processing of dielectrics.

In conclusion, fs pump-probe microscopy in bulk dielectrics is capable of providing a detailed and quantitative description of spatio-temporal properties of laser-induced transient plasmas under real processing conditions. We have identified filamentation and prefocal depletion as important energy loss channels in doped phosphate glass, which deteriorate the spatial distribution of the laser-deposited energy. By lowering the peak power, i.e., via pulse stretching, we obtained a well-defined spatial profile of the transient plasma, without energy being lost to filamentation. In addition, the use of circular laser polarization further minimizes filamentation due to larger $P_{\text {crit }}{ }^{o}$. Both strategies allow controlling the nonlinear propagation effects, paving the way for an efficient and optimized production of high quality and true three-dimensional photonic structures.

This work was partially supported by the Spanish Ministry of Science and Innovation under TEC 2005-00074 project and by the EU under TMR project FLASH (MRTNCT-2003-503641). W.G. acknowledges the I3P-CSIC contract (cofunded by the European Social Fund). D.P. and A.F. acknowledge their grants under Projects TEC 2005-00074 and TEC 2006-04538 and A. R. de la Cruz acknowledges his contract under FLASH Project. We acknowledge M. Galván for his help with the data acquisition software.

${ }^{1}$ Active Glass for Photonic Devices, edited by K. Hirao, T. Mitsuyu, J. Si, and J. Qiu (Springer, Berlin, Heidelberg, 2001).

${ }^{2}$ G. Della Valle, R. Osellame, G. Galzerano, N. Chiodo, G. Cerullo, P. Laporta, O. Svelto, U. Morgner, A. G. Rozhin, V. Scardaci, and A. C. Ferrari, Appl. Phys. Lett. 89, 231115 (2006)

${ }^{3}$ S. S. Mao, F. Quéré, S. Guizard, X. Mao, R. E. Russo, G. Petite, and P. Martin, Appl. Phys. A: Mater. Sci. Process. 79, 1695 (2004).

${ }^{4}$ R. W. Boyd, Nonlinear Optics (Academic, London, 2003).

${ }^{5}$ L. Sudrie, A. Couairon, M. Franco, B. Lamouroux, B. Prade, S. Tzortzakis, and A. Mysyrowicz, Phys. Rev. Lett. 89, 186601 (2002).

${ }^{6}$ K. Sokolowski-Tinten, J. Bialkowski, A. Cavalleri, D. von der Linde, A. Oparin, J. Meyerter-Vehn, and S. I. Anisimov, Phys. Rev. Lett. 81, 224 (1998).

${ }^{7}$ J. Siegel, D. Puerto, W. Gawelda, G. Bachelier, J. Solis, L. Ehrentraut, and J. Bonse, Appl. Phys. Lett. 91, 082902 (2007).

${ }^{8}$ Q. Sun, H. Jiang, Y. Liu, Z. Wu, H. Yang, and Q. Gong, Opt. Lett. 30, 320 (2005).

${ }^{9}$ V. Diez-Blanco, J. Siegel, A. Ferrer, A. Ruiz de la Cruz, and J. Solis, Appl. Phys. Lett. 91, 051104 (2007).

${ }^{10}$ A. Ferrer, V. Diez-Blanco, A. Ruiz, J. Siegel, and J. Solis, Appl. Surf. Sci. 254, 1121 (2007).

${ }^{11}$ M. Ams, G. D. Marshall, D. J. Spence, and M. J. Withford, Opt. Express 13, 5676 (2005).

${ }^{12}$ R. Stoian, M. Boyle, A. Thoss, A. Rosenfeld, G. Korn, and I. V. Hertel, Appl. Phys. Lett. 80, 353 (2002).

${ }^{13}$ L. Englert, B. Rethfeld, L. Haag, M. Wollenhaupt, C. Sarpe-Tudoran, and T. Baumert, Opt. Express 15, 17855 (2007).

${ }^{14}$ D. Puerto, W. Gawelda, J. Siegel, J. Bonse, G. Bachelier, and J. Solis, Appl. Phys. A: Mater. Sci. Process. 92, 803 (2008).

${ }^{15}$ A. Dubietis, G. Tamošauskas, G. Fibich, and B. Ilan, Opt. Lett. 29, 1126 (2004).

${ }^{16}$ D. M. Rayner, A. Naumov, and P. B. Corkum, Opt. Express 13, 3208 (2005).

${ }^{17}$ A. Pasquazi, S. Stivala, G. Assanto, J. Gonzalo, and J. Solis, Phys. Rev. A 77, 043808 (2008).

${ }^{18}$ R. L. Sutherland, Handbook of Nonlinear Optics (Marcel Dekker, New York, 2003).

${ }^{19}$ J. Gonzalo, H. Fernández, J. Solis, D. Muñoz-Martín, J. M. Fernández Navarro, C. N. Afonso, and J. L. G. Fierro, Appl. Phys. Lett. 90, 251907 (2007).

${ }^{20}$ V. V. Temnov, K. Sokolowski-Tinten, P. Zhou, A. El-Khamhawy, and D. von der Linde, Phys. Rev. Lett. 97, 237403 (2006).

${ }^{21}$ M. Ams, G. D. Marshall, and M. J. Withford, Opt. Express 14, 13158 (2006).

${ }^{22}$ Y. Shimotsuma, P. G. Kazansky, J. Qiu, and K. Hirao, Phys. Rev. Lett. 91, 247405 (2003).

${ }^{23}$ C. Hnatovsky, R. S. Taylor, E. Simova, P. P. Rajeev, D. M. Rayner, V. R. Bhardwaj, and P. B. Corkum, Appl. Phys. A: Mater. Sci. Process. 84, 47 (2006). 